\title{
Enlarged Lumen Volume of Proximal Aortic Segment and Acute Type B Aortic Dissection: A Computer Fluid Dynamics Study of Ideal Aortic Models
}

\author{
Yuan Peng' \\ Xuelan Zhang ${ }^{2}$ \\ Jiehua $\mathrm{Li}^{\prime}$ \\ Xiaolong Zhang' \\ $\mathrm{HaO} \mathrm{He}^{\mathrm{I}}$ \\ Xin $\mathrm{Li}^{1}$ \\ Kun Fang ${ }^{3}$ \\ Liancun Zheng ${ }^{2}$ \\ Chang Shu ${ }^{1,3}$ \\ 'Department of Vascular Surgery, Second \\ Xiangya Hospital, Central South \\ University, Changsha, 4I00I I, People's \\ Republic of China; ${ }^{2}$ School of \\ Mathematics and Physics, University of \\ Science and Technology Beijing, Beijing, \\ I00083, People's Republic of China; \\ ${ }^{3}$ Department of Vascular Surgery, Fuwai \\ Hospital, Chinese Academy of Medical \\ Science \& Peking Union Medical College, \\ Beijing, 100037, People's Republic of \\ China
}

Background: Recent study has revealed that enlarged diameters of the ascending aorta and proximal aortic arch enhance the probability of ATBAD. However, little is understood about the relation to ATBAD.

Objective: This study explored the differences in proximal aortic segment (PAS) morphology in patients with acute type B aortic dissection (ATBAD), and performed hemodynamic simulations to provide proof of principle.

Materials and Methods: The morphological characteristics of PAS in the ATBAD group $(n=163)$ and corresponding segment in the control group $(n=120)$ were retrospectively measured. The morphological parameters were analyzed using comprehensive statistical approaches. Ridge regression analysis was also performed to determine the association between independent variable and dependent variable. $\mathrm{P}<0.01$ was considered significant. Idealized aortic models were established based on variables of statistical significance, and hemodynamic simulations were performed to evaluate blood flow changes caused by morphology.

Results: Diameters at landmarks of PAS were significantly larger in the ATBAD group. The lumen volume $\left(\mathrm{V}_{\mathrm{PAS}}\right)$ of PAS in the ATBAD group was significantly enlarged than that of the control group $\left(124,659.07 \pm 34,089.27 \mathrm{~mm}^{3}\right.$ vs $\left.89,796.65 \pm 30,334.40 \mathrm{~mm}^{3} ; \mathrm{P}<0.001\right)$. Furthermore, the $\mathrm{V}_{\text {PAS }}$ was positively correlated to diameters. As the $\mathrm{V}_{\text {PAS }}$ increased, the fluid kinetic energy in PAS enhanced linearly, and time-averaged wall shear stress and oscillatory shear index at the distal area of the left subclavian artery increased significantly. Conclusion: In the ATBAD group, the enlarged $\mathrm{V}_{\text {PAS }}$ and increased diameters of PAS are positively correlated. Meanwhile, the enlarged $\mathrm{V}_{\text {PAS }}$ leads to more aggressive hemodynamic parameters at the distal area of the left subclavian artery, which may create a contributory condition for ATBAD.

Keywords: aortic dissection, aortic morphology, hemodynamics, ridge regression, kinetic energy

\section{Introduction}

Acute aortic dissection is a catastrophic aortic disease with an estimated incidence of 3.5 per 100.000 persons per year. ${ }^{1}$ Acute type B aortic dissection (ATBAD) accounts for $25 \%$ to $40 \%$ of all aortic dissections. ${ }^{2}$ The onset of the disease is sudden, which makes it difficult to predict, and thus difficult to prevent. Research has indicated that changes in aortic morphology are related to aortic dissection, such as that the maximum diameter of the aorta greater than $5.5 \mathrm{~cm}$ dramatically increases the risk of aortic dissections. ${ }^{3}$ But the maximum diameter of the
Correspondence: Chang Shu

Tel +86-73I-85295832

Email shuchang@csu.edu.cn 
descending aorta greater than or equal to $5.5 \mathrm{~cm}$ is not an accurate predictor of ATBAD, ${ }^{4}$ and a considerable number of patients present ATBAD in the absence of aortic dilatation. ${ }^{5}$ Therefore, identifying more morphologic risk factors may help to better identify patients at risk for ATBAD. Recent study has revealed that enlarged diameters of the ascending aorta and proximal aortic arch enhance the probability of ATBAD. ${ }^{6,7}$ However, little is understood about the relation to ATBAD.

Hemodynamic simulation has been widely used in the study of aortic diseases, ${ }^{8-11}$ and has enabled the quantification of the specific impact of each aortic anatomical risk factor. ${ }^{12}$ Polanczyk et al created a mathematical approach for blood hemodynamic description with the use of brightness analysis and proved post-operative remodelling of the aorta after TEVAR for aortic dissection improved hemodynamic patterns. These examples open up a whole new perspective on the clinical application of computational fluid dynamics. ${ }^{13,14}$ In an attempt to provide a new perspective for the evolution of ATBAD, our study aims to thoroughly understand the relationship between aortic morphology and ATBAD. Unlike previous studies, we focus on the influence of upstream aortic morphology changes on downstream aortic hemodynamic characteristics. The process of blood flow in the aorta is also the process by which the heart assigns fluid energy to each artery. The fluid energy of the bloodstream in the aorta follows the principle of conservation of mass and momentum, so the fluid energy in the downstream aorta depends on that of the upstream fluid.

Our aim is to explore the differences in aortic morphology in patients with ATBAD. To understand the relationship between aortic anatomy and ATBAD, several idealized aortic design models based on actual aortic morphological differences were developed, and hemodynamic simulations were performed to provide proof of the principle of the conservation of mass and momentum.

\section{Materials and Methods Study Design}

In this retrospective study, patients diagnosed with ATBAD (onset not more than two weeks) at the Second Xiangya Hospital between April 2018 and July 2019 were analyzed. Exclusion criteria were as follows: 1) patients without available computed tomography angiography (CTA) scans or with poor-quality scans; 2) patients with known connective tissue disorders; 3) patients with iatrogenic or traumatic dissection, and chronic aortic dissection; 4) patients $<30$ years old; 5) patients with congenital aortic malformations; 6) patients with congenital anomalies of the aortic arch branch; 7) patients had undergone previous aortic surgery; 8) aortic arch or left subclavian artery involved hematomas. Finally, 163 patients were incorporated into the ATBAD group. The control group consisted of patients who received CTA for a non-vascular emergency at the Second Xiangya Hospital between April 2018 and June $2018(\mathrm{n}=184)$. Expectedly, these included fairly young adults. For homogenization, we excluded all patients below the age of 30 from this group $(n=64)$, as a result, 120 patients were incorporated into the control group. Demographic data including age and sex were collected. This retrospective study was approved by the Medical Ethics Committee of the Second Xiangya Hospital of Central South University (NO.2020S017), and the need for informed consent was waived. It was conducted following the Declaration of Helsinki. Information of the patients for this study was kept strictly confidential.

\section{CTA Scans}

CTA scans were carried out at the Second Xiangya Hospital using a third-generation dual-source CT scanner (SOMATOM Definition Force, Siemens Healthcare, Forchheim, Germany), with a high iodinated contrast bolus of $100 \mathrm{~mL}(370 \mathrm{mg} / \mathrm{mL}$ iodine). The bolus was injected at a rate of $4-5 \mathrm{~mL} / \mathrm{s}$ and was chased by saline. CTA images with a maximum slice thickness of $1 \mathrm{~mm}$ were accepted for further processing.

\section{Aortic Landmarks and Image Processing}

CTA datasets were processed using MIMICS (v20.0, Materialise, Belgium) software to reconstruct the 3D geometrical models of the aortas (Figure 1). The reconstructed arteries were subsequently smoothed with the Laplacian smoothing method to diminish noises caused by the discontinuity of image data or uncertainties in lumen boundary identification. The cross-sectional contours of the reconstructed geometries were mapped back to CTA images to ensure that the $3 \mathrm{D}$ models present the actual outline of the vessel lumen. Then, the centerline of the aorta (excluding the three branches on the arch, the branches below the iliac artery, the renal artery and other small branches) was extracted. The aortic segment from the aortic valve annulus to distal of the left subclavian artery orifice was defined as the proximal aortic segment (PAS). The following landmarks were applied for assessing the equivalent diameter of the aortic segment 

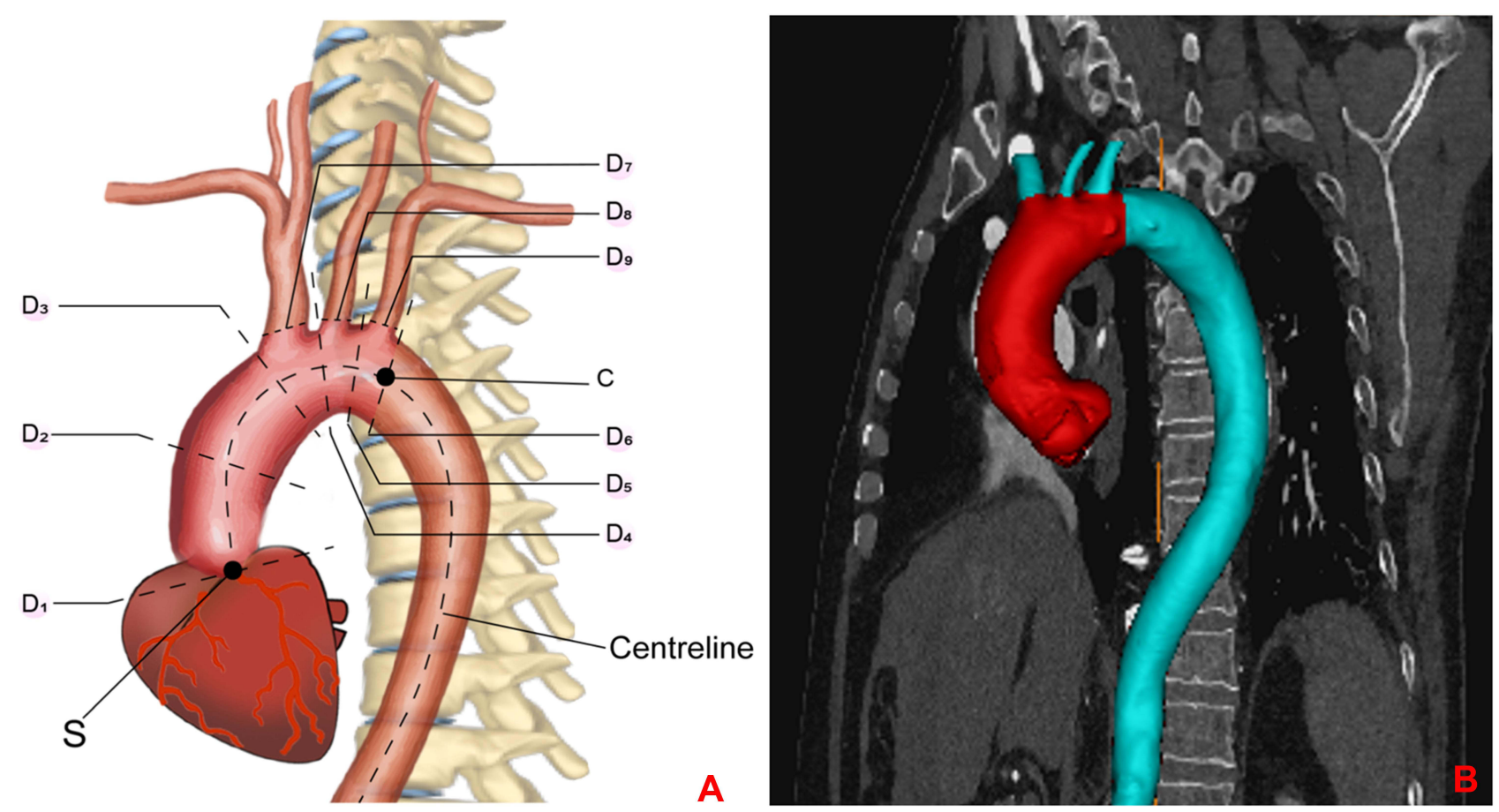

Figure I (A) Aortic landmarks: $D_{1}$, aortic valve annulus; $D_{2}$, mid-ascending aorta; $D_{3}$, proximal of the brachiocephalic trunk orifice; $D_{4}$, proximal of the left common carotid artery orifice; $D_{5}$, proximal of the left subclavian artery orifice; $D_{6}$, distal of the left subclavian artery orifice; $D_{7}$, orifice of the brachiocephalic trunk; $D_{8}$, orifice of the left common carotid artery; $\mathrm{D}_{9}$, orifice of the left subclavian artery; $\mathrm{S}$, the intersection of the centerline with the aortic valve annulus plane; $\mathrm{C}$, the intersection of the centerline with distal of subclavian artery plane. (B) Red area stands for proximal aortic segment.

$\left(D=2 \times \sqrt{\frac{M}{\pi}}, \mathrm{M}\right.$ stands for the cross-section area): $\mathrm{D}_{1}$, aortic valve annulus; $\mathrm{D}_{2}$, mid-ascending aorta; $\mathrm{D}_{3}$, proximal of the brachiocephalic trunk orifice; $\mathrm{D}_{4}$, proximal of the left common carotid artery orifice; $\mathrm{D}_{5}$, proximal of the left subclavian artery orifice; $\mathrm{D}_{6}$, distal of the left subclavian artery orifice; $\mathrm{D}_{7}$, orifice of the brachiocephalic trunk; $\mathrm{D}_{8}$, orifice of the left common carotid artery; $\mathrm{D}_{9}$, orifice of the left subclavian artery. The length measurement of the aortic segments was defined as follows: L, length of PAS. The marker nodes on the centerline were defined as follows: $\mathrm{S}$, the intersection of the centerline with the aortic valve annulus plane; $\mathrm{C}$, the intersection of the centerline with distal of the subclavian artery plane. The tortuosity was defined as the ratio of the length of the centerline to the straight-line distance between two points: $\mathrm{T}$, tortuosity of PAS. $\mathrm{V}_{\mathrm{PAS}}$, lumen volume of PAS.

\section{Evaluation of Interobserver Image Measurement Consistency}

To assess inter-class reliability, two vascular surgeons took measurements simultaneously in the same protocol framework. Inter-class correlation coefficient (ICC) was used to evaluate the consistency of the image geometry features.
ICC $>0.75$ indicates that this measuring result had good consistency.

\section{Hemodynamic Simulation and Data Quantification Construction of Ideal Model}

Seven idealized aortic computer aided design models featuring the morphology of the aorta and the three branches of the aortic arch, ie, left subclavian, left common carotid, and brachiocephalic arteries were established to evaluate the overall kinetic energy of the bloodstream in this segment. This approach enabled a controlled comparison among models, as only one geometrical factor ( $\left.\mathrm{V}_{\text {PAS }}\right)$ differed and branches were constant and the descending aorta was far from the left subclavian artery. The varying $V_{\text {PAS }}$ is realized by fixing $D_{6}$ and adjusting $D_{1}-D_{5}$ in different degrees. At the same time, the adjustment proportion of $\mathrm{D}_{1}-\mathrm{D}_{5}$ is consistent. $\mathrm{V}_{\text {PAS }}$ increases from $1.15 \mathrm{E}$ $+05 \mathrm{~mm}^{3}$ to $1.53 \mathrm{E}+05 \mathrm{~mm}^{3}$; the maximal increased magnitude of $\mathrm{V}_{\text {PAS }}$ was approximately $33 \%$.

\section{Mesh and Time-Step Independence Tests}

Four unstructured meshes consisting of 1.1-1.8 million hexahedral elements with ten prismatic layers, reducing 
in size towards the wall, to ensure adequate near wall resolution were generated with ANSYS ICEM 19.0 (ANSYS, Inc., Canonsburg, PA, USA). These meshes were tested as part of a sensitivity analysis, and the chosen mesh (with about 1.6 million elements) differed from the finer mesh in the predicted maximum and minimum results by less than $2 \%$. Three time step settings were $0.0005 \mathrm{~s}$, $0.001 \mathrm{~s}$ and $0.005 \mathrm{~s}$. Reducing the time step from 0.005 $\mathrm{s}$ to $0.0005 \mathrm{~s}$ leads to a small discrepancy in the velocity and pressure distribution (less than 1\%), but significantly increases the computational cost, so a time step of 0.001 $\mathrm{s}$ is used in the present study. Simulation was carried out for 5 cardiac cycles (total 4 seconds) to achieve a periodic solution, and results of the final cycle are presented. The computational simulation was calculated using ANSYS FLUENT 19.0 (ANSYS, Inc., Canonsburg, PA, USA).

\section{Boundary Conditions and Blood Property}

A transient parabolic flow inlet $\mathrm{Q}(\mathrm{t})$ and a pulsatile pressure waveform outlet $\mathrm{p}(\mathrm{t})$ are applied at the ascending aorta and the descending aorta, respectively. ${ }^{15}$ Based on previous studies, 5\% of the flow volume is diverted to each branch outlet, ie, left subclavian artery, left common carotid artery, and brachiocephalic artery. ${ }^{16}$ The aortic wall is assumed to be rigid according to the vivo data that show low distensibility in long-term follow-ups, ${ }^{17}$ and no-slip and non-porous boundary conditions are designated at the aortic wall. The blood was modeled as incompressible non-Newtonian fluid. As the Reynolds number less than 2000 in peak systolic phase, we analysed the laminar flow analysis following the Carreau-Yasuda model, which fits the experimental data of the blood at $37.0^{\circ} \mathrm{C},{ }^{12,18}$ and the density of the blood was $1060 \mathrm{~kg} / \mathrm{m}^{3}$. ${ }^{17,19}$

\section{Statistical Analysis}

Statistical analysis was performed using SPSS 20.0 software (IBM, Armonk, NY, USA) and MedCalc 18.2.1 software (Mariakerke, Belgium). The distribution of continuous variables was assessed for normality using the Shapiro-Wilk test. Categorical variables are presented as counts, and continuous variables were presented as mean \pm standard deviation (SD). Differences between the study groups were tested for significance using Student $t$ test, Mann Whitney $U$-test or $\chi^{2}$ test. Spearman correlation analysis was performed to explore the correlations among variables and variance inflation factor (VIF) was calculated to assess the collinearity of independent variables. Ridge regression analysis was also performed to determine the association between independent variable and dependent variable. Receiver-operating characteristic (ROC) curves were used to assess the diagnostic value. All reported $\mathrm{P}$ values were two sided; $\mathrm{P}<0.01$ was considered significant.

\section{Results}

\section{Baseline Characteristics and Morphological Distinction}

Table 1 shows that the prevalence of hypertension was higher in the ATBAD group. There were no significant differences in age, gender, body mass index (BMI) and smoking between the control group and the ATBAD group.

Table 2 shows the diameters of different aortic segments and branches of the aortic arch included in the study groups. The diameters of the aortic arch branches were not significantly different between the control group and the ATBAD group, neither were the length and tortuosity of PAS. All

Table I Baseline Characteristics of Patients

\begin{tabular}{|l|c|c|c|}
\hline & Control $(\mathbf{n}=\mathbf{I 2 0})$ & ATBAD $(\mathbf{n}=\mathbf{1 6 3})$ & P value \\
\hline Age $(\mathrm{y})$ & $56.21 \pm 12.62$ & $55.42 \pm 11.00$ & 0.813 \\
Gender $(\mathrm{M} / \mathrm{F})$ & $82 / 38$ & $119 / 44$ & 0.473 \\
BMI $\left(\mathrm{kg} / \mathrm{m}^{2}\right)$ & $26.5 \pm 3.2$ & $26.2 \pm 3.7$ & 0.304 \\
Hypertension & $42.5 \%$ & $84.7 \%$ & $<0.001$ \\
Smoking & $65 \%$ & $67 \%$ & 0.275 \\
\hline
\end{tabular}

Abbreviation: BMI, body mass index.

Table 2 Geometrical Parameters Included in the Analysis

\begin{tabular}{|c|c|c|c|}
\hline Variable & Control $(n=120)$ & ATBAD $(n=163)$ & $P$ value \\
\hline $\mathrm{D}_{\mathrm{I}}(\mathrm{mm})$ & $30.55 \pm 3.83$ & $34.60 \pm 4.69$ & $<0.001$ \\
\hline $\mathrm{D}_{2}(\mathrm{~mm})$ & $34.29 \pm 3.83$ & $38.08 \pm 4.17$ & $<0.001$ \\
\hline $\mathrm{D}_{3}(\mathrm{~mm})$ & $32.42 \pm 3.72$ & $35.74 \pm 3.40$ & $<0.001$ \\
\hline $\mathrm{D}_{4}(\mathrm{~mm})$ & $30.15 \pm 3.48$ & $33.68 \pm 3.27$ & $<0.001$ \\
\hline $\mathrm{D}_{5}(\mathrm{~mm})$ & $28.31 \pm 2.98$ & $31.41 \pm 3.18$ & $<0.001$ \\
\hline $\mathrm{D}_{6}(\mathrm{~mm})$ & $27.23 \pm 2.91$ & $30.36 \pm 3.23$ & $<0.001$ \\
\hline $\mathrm{D}_{7}(\mathrm{~mm})$ & $15.01 \pm 2.81$ & $16.48 \pm 2.65$ & 0.091 \\
\hline $\mathrm{D}_{8}(\mathrm{~mm})$ & $10.50 \pm 2.03$ & $\mid 1.43 \pm 3.41$ & 0.082 \\
\hline $\mathrm{D}_{9}(\mathrm{~mm})$ & $11.80 \pm 2.48$ & $12.88 \pm 2.00$ & 0.103 \\
\hline $\mathrm{L}(\mathrm{mm})$ & $112.11 \pm 14.73$ & $115.39 \pm 12.30$ & 0.120 \\
\hline $\mathrm{T}$ & $1.33 \pm 0.09$ & $1.31 \pm 0.24$ & 0.483 \\
\hline$V_{\text {PAS }}\left(\mathrm{mm}^{3}\right)$ & $89,796.65 \pm 30,334.40$ & $124,659.07 \pm 34,089.27$ & $<0.001$ \\
\hline
\end{tabular}

Abbreviations: $D_{1}$, aortic valve annulus diameter; $D_{2}$, mid-ascending aorta diameter; $D_{3}$, aorta diameter at proximal of the brachiocephalic trunk orifice; $D_{4}$, aorta diameter at proximal of the left common carotid artery orifice; $D_{5}$, aorta diameter at proximal of the left subclavian artery orifice; $D_{6}$, aorta diameter at distal of the left subclavian artery orifice; $D_{7}$, the brachiocephalic trunk orifice diameter; $D_{8}$, the left common carotid artery orifice diameter; $D_{9}$, the left subclavian artery orifice diameter; $L$, length of proximal aortic segment; $T$, tortuosity of proximal aortic segment; $\mathrm{V}_{\mathrm{PAS}}$, lumen volume of proximal aortic segment. 


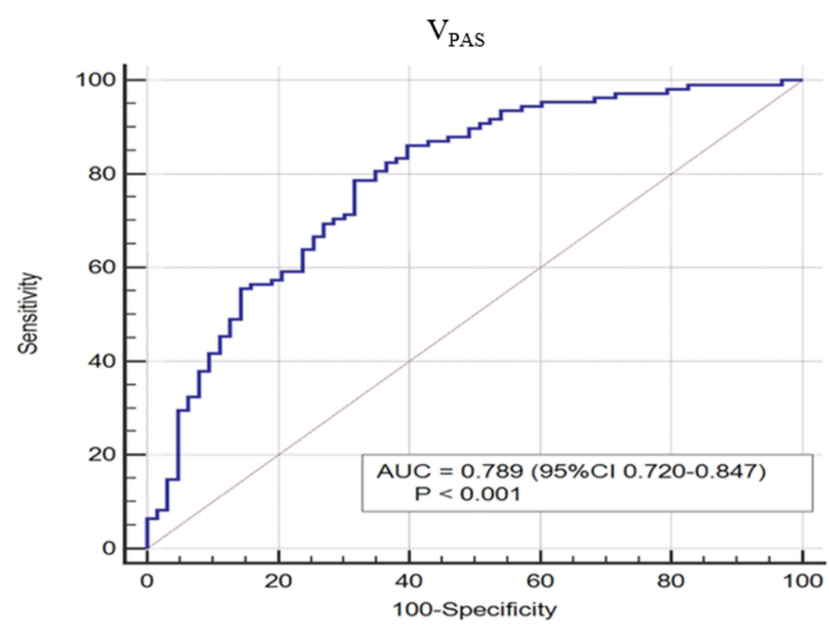

Figure 2 Receiver-operating curve for lumen volume of proximal aortic segment $\left(\mathrm{V}_{\mathrm{PAS}}\right)$

Abbreviation: AUC, the area under the curve.

diameters of PAS in the ATBAD group were significantly ( $\mathrm{P}<$ 0.001) larger than those in the control group. The $\mathrm{V}_{\mathrm{PAS}}$ in the ATBAD group significantly exceeded that of the control group $\left(124,659.07 \pm 34,089.27 \mathrm{~mm}^{3}\right.$ vs $89,796.65 \pm 30,334.40 \mathrm{~mm}^{3}$, $\mathrm{P}<0.001$ ). The $\mathrm{V}_{\mathrm{PAS}}$ had an area under the curve (AUC) of 0.789 (95\% CI: 0.720 to $0.847 ; \mathrm{P}<0.001$ ) (Figure 2).

\section{Correlation Between the Diameter of Different Aortic Segments and V in Patients}

The correlation of $\mathrm{V}_{\mathrm{PAS}}$ with the diameter of aortic segments is summarized in Table 3. $\mathrm{V}_{\mathrm{PAS}}$ of different patient groups were positively correlated to $\mathrm{D}_{1}, \mathrm{D}_{2}, \mathrm{D}_{3}, \mathrm{D}_{4}, \mathrm{D}_{5}, \mathrm{D}_{6}(\mathrm{r}=0.64,0.79$, $0.71,0.66,0.64,0.63$, respectively, $\mathrm{P}<0.001)$. Multiple regression analysis showed that there was a significant correlation among the variables. Collinearity diagnostic tests revealed severe collinearity among the independent variables (VIF:
$\left.D_{3}=9.88, D_{4}=11.64, D_{5}=10.43\right)$. The ridge regression analysis adjusted for hypertension was further used to calibrate (Figure 3). The parameters were estimated with $\mathrm{k}=0.6$, when the standardized regression coefficients of each independent variable were stable. The results indicated that $\mathrm{D}_{1}, \mathrm{D}_{2}, \mathrm{D}_{3}, \mathrm{D}_{4}$, $\mathrm{D}_{5}, \mathrm{D}_{6}$ were independent factors associated with $\mathrm{V}_{\mathrm{PAS}}$ ( $\beta=0.64,0.79,0.71,0.66,0.64,0.63$, respectively, $\mathrm{P}<0.01$ ) (Table 4).

\section{Hemodynamic Analysis}

As depicted in the pathlines diagram of Figure 4, idealized models were established to simulate several hemodynamic conditions with varying morphological characteristics in Figure 4A. The inlet flow curve and outlet pressure curve are shown in Figure 4B-b and Figure 4B-c, a vortex was observed in PAS that was extracted as the region of interest. Vortex generation moved the blood away from the aortic wall towards the center of the aorta, which had direct effects on the fluid kinetic energy. Numerical simulations were performed to investigate the variations in the kinetic energy using qualitative and quantitative analyses methods (Figure 4C-b). The streamline diagrams show that the flow velocity of blood is gradually enhanced with the increasing $\mathrm{V}_{\mathrm{PAS}}$. The kinetic energy in the diagram of curves represent the overall energy throughout the cardiac cycle. As the $\mathrm{V}_{\mathrm{PAS}}$ increases from $1.15 \mathrm{E}+05 \mathrm{~mm}^{3}$ to $1.53 \mathrm{E}$ $+05 \mathrm{~mm}^{3}$, it can be seen that the increased magnitude of fluid kinetic energy is approximately $22.3 \%$. The fluid kinetic energy is positively, linearly correlated with the $\mathrm{V}_{\text {PAS }}$. Computational fluid dynamics simulation shows there is a significant relationship between $\mathrm{V}_{\mathrm{PAS}}$ and hemodynamic factors at the distal area of the left subclavian artery (Figure 4C-c). The time-averaged wall shear stress (TAWSS) and oscillatory shear index (OSI) in the diagram

Table 3 Correlation Analysis and Multiple Linear Regression Analysis Between Lumen

\begin{tabular}{|c|c|c|c|c|c|c|c|c|c|}
\hline \multirow{2}{*}{$\begin{array}{l}\text { Dependent } \\
\text { Variable }\end{array}$} & \multirow{2}{*}{$\begin{array}{l}\text { Independent } \\
\text { Variables }\end{array}$} & \multicolumn{2}{|c|}{ Spearman Correlation } & \multicolumn{6}{|c|}{ Multiple Linear Regression } \\
\hline & & $\mathbf{r}$ & $\mathbf{P}$ & $\boldsymbol{\beta}$ & SE & $\mathbf{t}$ & $\mathbf{P}$ & Tolerance & VIF \\
\hline \multirow[t]{6}{*}{$V_{\text {PAS }}$} & $D_{1}$ & 0.64 & $<0.01$ & 1.3 & 0.50 & 2.7 & $<0.01$ & 0.48 & 2.1 \\
\hline & $D_{2}$ & 0.79 & $<0.01$ & 5.9 & 0.92 & 6.4 & $<0.01$ & 0.16 & 6.3 \\
\hline & $\mathrm{D}_{3}$ & 0.71 & $<0.01$ & -2.3 & 1.32 & -1.7 & 0.09 & 0.10 & 9.9 \\
\hline & $\mathrm{D}_{4}$ & 0.66 & $<0.01$ & 0.7 & 1.47 & 0.5 & 0.64 & 0.09 & 11.6 \\
\hline & $D_{5}$ & 0.64 & $<0.01$ & 0.6 & 1.50 & 0.4 & 0.70 & 0.10 & 10.4 \\
\hline & $D_{6}$ & 0.63 & $<0.01$ & 1.1 & 0.99 & 1.1 & 0.28 & 0.23 & 4.4 \\
\hline
\end{tabular}

Note: Volume of proximal segment $\left(V_{P A S}\right)$ and diameter of aortic segments $\left(D_{1}-D_{6}\right)$.

Abbreviations: $V_{P A S}$, lumen volume of proximal aortic segment; $D_{1}$, aortic valve annulus diameter; $D_{2}$, mid-ascending aorta diameter; $D_{3}$, aorta diameter at proximal of the brachiocephalic trunk orifice; $\mathrm{D}_{4}$, aorta diameter at proximal of the left common carotid artery orifice; $\mathrm{D}_{5}$, aorta diameter at proximal of the left subclavian artery orifice; $D_{6}$, aorta diameter at distal of the left subclavian artery orifice. 


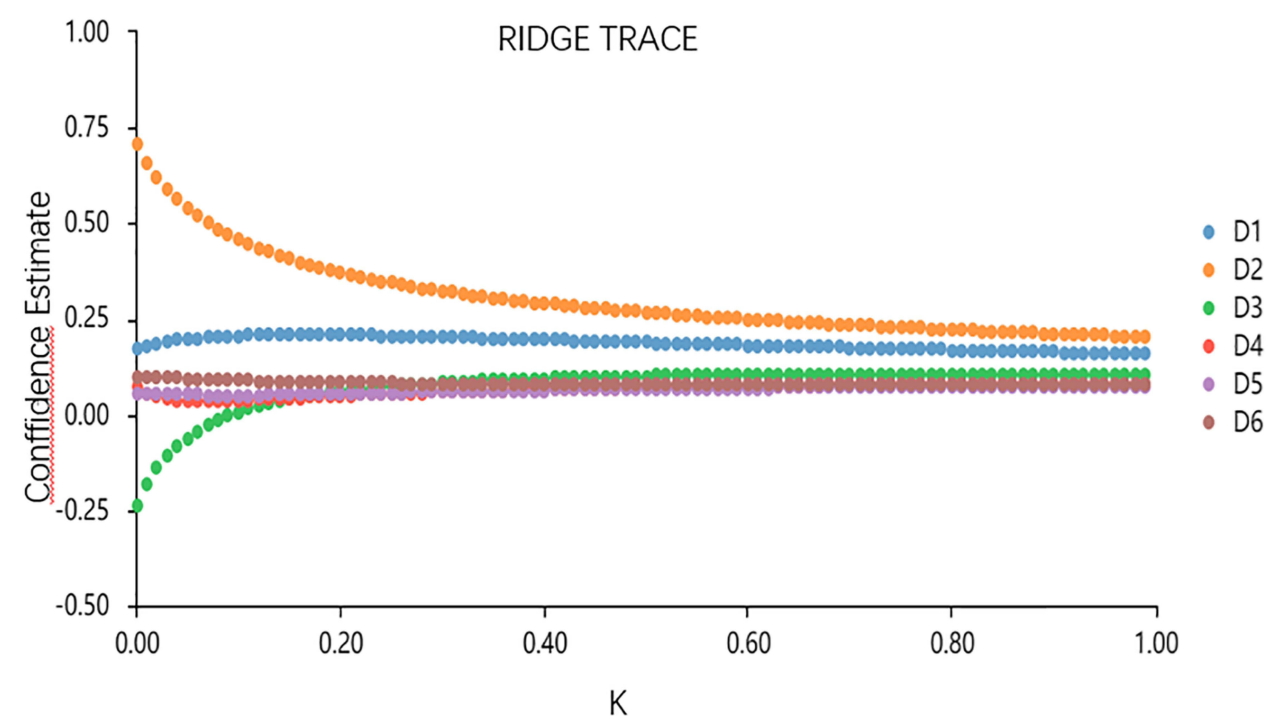

Figure 3 Ridge trace curve of the association between the lumen volume ( $\left.\mathrm{V}_{\mathrm{PAS}}\right)$ and diameters at landmark (D) in proximal aortic segment.

of curves represent the cardiac cycle area average value. As the $\mathrm{V}_{\mathrm{PAS}}$ increases from $1.15 \mathrm{E}+05 \mathrm{~mm}^{3}$ to $1.53 \mathrm{E}$ $+05 \mathrm{~mm}^{3}$, the TAWSS and OSI increase about 2 times.

\section{Discussion}

This study focused on the morphology and hemodynamic characteristics of PAS, to explore the interrelationships of PAS morphology, hemodynamics, and ATBAD. Result shows: 1) diameters at all landmarks of PAS and the $\mathrm{V}_{\mathrm{PAS}}$ in the ATBAD group were significantly increased compared with the control group, and the $\mathrm{V}_{\mathrm{PAS}}$ of different patient groups was positively correlated to diameters at all landmarks of PAS; 2) as the $\mathrm{V}_{\mathrm{PAS}}$ increased, the flow

Table 4 Ridge Regression Analysis of Lumen Volume of Proximal Non-Dissection Aortic Segment ( $\left.V_{\mathrm{PAS}}\right)$ and Diameter of Aortic Segments $\left(D_{1}-D_{6}\right)$

\begin{tabular}{|l|c|c|c|c|}
\hline \multirow{2}{*}{} & \multicolumn{4}{|c|}{ VPAS } \\
\cline { 2 - 5 } & $\boldsymbol{\beta}$ & $\mathbf{S E}(\boldsymbol{\beta})$ & $\mathbf{t}$ & $\mathbf{P}$ \\
\hline Constant & -117.76 & $14.5 \mathrm{I}$ & -8.12 & $<0.0 \mathrm{I}$ \\
$\mathrm{D}_{1}$ & 1.40 & 0.22 & 6.34 & $<0.0 \mathrm{I}$ \\
$\mathrm{D}_{2}$ & 2.06 & 0.20 & 10.40 & $<0.0 \mathrm{I}$ \\
$\mathrm{D}_{3}$ & 0.97 & 0.20 & 4.79 & $<0.0 \mathrm{I}$ \\
$\mathrm{D}_{4}$ & 0.72 & 0.20 & 3.63 & $<0.0 \mathrm{I}$ \\
$\mathrm{D}_{5}$ & 0.75 & 0.23 & 3.37 & $<0.0 \mathrm{I}$ \\
$\mathrm{D}_{6}$ & 0.846 & 0.27 & $3.1 \mathrm{I}$ & $<0.0 \mathrm{I}$ \\
\hline
\end{tabular}

Abbreviations: $V_{\mathrm{PAS}}$, lumen volume of proximal aortic segment; $D_{1}$, aortic valve annulus diameter; $D_{2}$, mid-ascending aorta diameter; $D_{3}$, aorta diameter at proximal of the brachiocephalic trunk orifice; $\mathrm{D}_{4}$, aorta diameter at proximal of the left common carotid artery orifice; $D_{5}$, aorta diameter at proximal of the left subclavian artery orifice; $D_{6}$, aorta diameter at distal of the left subclavian artery orifice. velocity of the blood was gradually enhanced, the fluid kinetic energy increased linearly, and the time-averaged wall shear stress (TAWSS) and oscillatory shear index (OSI) at the immediate area distal to the orifice of the left subclavian artery increased significantly.

Numerous studies have revealed that the dilation of the aorta is age and gender dependent, whereas correlations with BMI and smoking are weak. ${ }^{3,20,21}$ In the sample, all these factors were equally distributed in both study groups. More than $80 \%$ of the studied patients who developed acute type B aortic dissection had hypertension, which coincided with previous studies. ${ }^{7}$ Hypertension can place increased pressure on the aortic wall, which may have an association with the development of aortic sections. ${ }^{3,22}$

The diameters of PAS in the ATBAD group were larger than those of the control group in this study, which was consistent with previous studies. ${ }^{6,7}$ The increased $V_{\text {PAS }}$ was proved to be significantly associated with an increased risk for ATBAD, and exhibited a superior diagnostic value, with a ROC-AUC of 0.789 . Meanwhile, several studies have revealed that the $\mathrm{V}_{\mathrm{PAS}}$ improved the sensitivity for detection of morphological changes compared with diameter measurements. ${ }^{23,24}$ The formula for $\mathrm{V}_{\mathrm{PAS}}$ shows that the $\mathrm{V}_{\mathrm{PAS}}$ is related to the diameter and length of the PAS. Univariate contrasts found no statistically significant differences in L between the two groups, which suggests that the increase in the $\mathrm{V}_{\mathrm{PAS}}$ is due to the dilatation of PAS in the ATBAD group. Spearman correlation analysis demonstrated a correlation between $V_{P A S}$ and diameters $\left(D_{1}-D_{6}\right)$, and collinearity diagnostic tests indicated a collinearity 


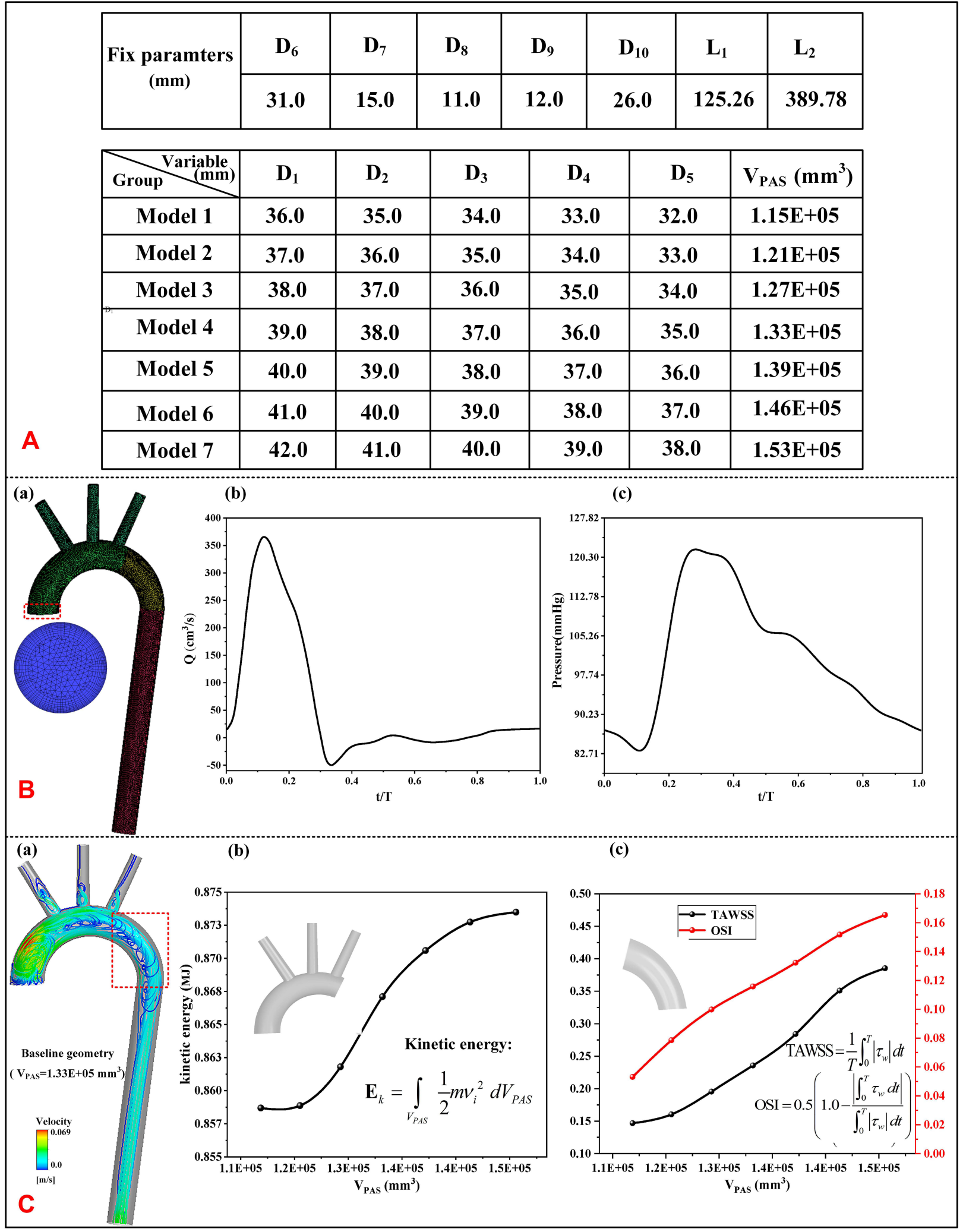

Figure 4 (A) Detail parameters for ideal models. $D_{1}$, aortic valve annulus diameter; $D_{2}$, mid-ascending aorta diameter; $D_{3}$, aorta diameter at proximal of the brachiocephalic trunk orifice; $D_{4}$, aorta diameter at proximal of the left common carotid artery orifice; $D_{5}$, aorta diameter at proximal of the left subclavian artery orifice; $D_{6}$, aorta diameter at distal of the left subclavian artery orifice; $D_{7}$, the brachiocephalic trunk orifice diameter; $D_{8}$, the left common carotid artery orifice diameter; $D_{9}$, the left subclavian artery orifice diameter; $\mathrm{D}_{10}$, diameter of distal descending aorta; $\mathrm{LI}$, length of proximal aortic segment; $\mathrm{L}_{2}$, length of descending aorta; $\mathrm{V}_{\mathrm{PAS}}$, lumen volume of proximal aortic segment. (B-a) Creation of polyhedral meshes; (B-b) Inlet flow curve; (B-c) Outlet pressure curve. (C-a) Streamlines for the baseline geometry; (C-b) The variations of kinetic energy versus the $\mathrm{V}_{\mathrm{PAS}}$; (C-c) TAWSS and OSI versus $\mathrm{V}_{\mathrm{PAS}}$.

Abbreviations: TAWSS, time-averaged wall shear stress; OSI, oscillatory shear index. 
among the independent variables $\left(D_{1}-D_{6}\right)$. When there is an issue of collinearity among independent variables, the regression coefficients obtained from traditional multiple linear regression analysis and stepwise regression analysis lack precision and the results may be distorted or even contradictory to the facts. Ridge regression is an improved least-squares regression analysis and is more applicable to the case of collinearity between independent variables. ${ }^{25-27}$ Adjusted for hypertension, the effects of the independent variables were determined by ridge regression analysis.

What is the relationship between the increased $\mathrm{V}_{\mathrm{PAS}}$ and ATBAD? To answer this question, a series of ideal models was established based on the discrepancy of the spatial geometry of PAS and hemodynamic simulations were performed. The flow velocity of blood and the fluid kinetic energy of PAS enhanced as the $\mathrm{V}_{\text {PAS }}$ increased. The greater the kinetic energy of the upstream fluid, the greater the force applied to the downstream vascular wall. Relatively high TAWSS and OSI at the distal area of the left subclavian artery may be associated with tear initiation and propagation. ${ }^{15,28}$ According to the principle of biological stress balance of the aortic wall, ${ }^{29}$ this could be a contributory factor for ATBAD. From the perspective of fluid mechanics, the incremental blood pressure increase leads to an increase in the fluid kinetic energy. ${ }^{30}$ Therefore, the increase in fluid kinetic energy caused by the increased $\mathrm{V}_{\mathrm{PAS}}$ in this study had an effect similar to hypertension, contributing to ATBAD.

\section{Conclusion}

All diameters of PAS in the ATBAD group were significantly $(\mathrm{P}<0.001)$ larger than those in the control group. The VPAS in the ATBAD group significantly exceeded that of the control group $\left(124,659.07 \pm 34,089.27 \mathrm{~mm}^{3}\right.$ vs $89,796.65 \pm$ $\left.30,334.40 \mathrm{~mm}^{3}, \mathrm{P}<0.001\right)$. In the ATBAD group, the enlarged $V_{\text {PAS }}$ and increased diameters of PAS are positively correlated. As the $\mathrm{V}_{\text {PAS }}$ increases from $1.15 \mathrm{E}+05 \mathrm{~mm}^{3}$ to $1.53 \mathrm{E}+05 \mathrm{~mm}^{3}$, the TAWSS and OSI increase about 2 times. The enlarged $V_{\text {PAS }}$ leads to more aggressive hemodynamic parameters at the distal area of the left subclavian artery, which may create a contributory condition for ATBAD.

\section{Study Strengths and Limitations}

This was a retrospective cross-sectional study combining morphological analysis and hemodynamic simulation. It was the first time to explore how the increased volume of the ascending aorta and aortic arch could promote ATBAD from the perspective of kinetic energy transport.
It has been demonstrated that rigid wall analysis overpredicts the pulsatility of both pressure and flow waveforms, and overpredicts will be larger in the more compliant aortic model. ${ }^{12}$ We could not replicate physiologic phase shifts and attenuation of outflow using a rigid-wall assumption. ${ }^{31}$ All results are under hypothetical conditions, all ideal models can only partially explain the possible principles. It is not easy to assess the individual impact of an anatomical factor that plays a role in ATBAD. However, our simple analysis method can capture the changing trend of some characteristics of the flow field under our hypothetical conditions. It is through the study of simplified models that the relative value of the anatomical variation can be understood.

The single-center retrospective approach limited the application of the relationship of $\mathrm{V}_{\mathrm{PAS}}$ and diameters in PAS and the $\mathrm{V}_{\mathrm{PAS}}$ as a predictor for ATBAD. Nevertheless, the findings of significant discrepancies in morphology and morphology-driven hemodynamics in PAS were meaningful to thoroughly understand the formation mechanism of ATBAD.

\section{Funding}

This research was supported by the National Natural Science Foundation of China (81870345).

\section{Disclosure}

The authors declare no potential conflicts of interest with respect to the research, authorship, and/or publication of this article.

\section{References}

1. Nienaber CA, Clough RE, Sakalihasan N, et al. Aortic dissection. Nat Rev Dis Primers. 2016;2:16053. doi:10.1038/nrdp.2016.53

2. Hughes GC. Management of acute type B aortic dissection; ADSORB trial. J Thorac Cardiovasc Surg. 2015;149(2 Suppl):S158-62. doi:10.1016/j.jtcvs.2014.08.083

3. Erbel R, Aboyans V, Boileau C, et al. 2014 ESC Guidelines on the diagnosis and treatment of aortic diseases: document covering acute and chronic aortic diseases of the thoracic and abdominal aorta of the adult. The Task Force for the Diagnosis and Treatment of Aortic Diseases of the European Society of Cardiology (ESC). Eur Heart J. 2014;35(41):2873-2926. doi:10.1093/eurheartj/ehu281

4. Trimarchi S, Jonker FH, Hutchison S, et al. Descending aortic diameter of $5.5 \mathrm{~cm}$ or greater is not an accurate predictor of acute type B aortic dissection. J Thorac Cardiovasc Surg. 2011;142(3):e101-7. doi:10.1016/j.jtcvs.2010.12.032

5. Trimarchi S, Jonker FH, Froehlich JB, et al. Acute type B aortic dissection in the absence of aortic dilatation. J Vasc Surg. 2012;56 (2):311-316. doi:10.1016/j.jvs.2012.01.055

6. Lescan M, Veseli K, Oikonomou A, et al. Aortic elongation and Stanford B dissection: the Tubingen Aortic Pathoanatomy (TAIPAN) project. Eur J Vasc Endovasc Surg. 2017;54(2):164-169. doi:10.1016/ j.ejvs.2017.05.017 
7. Shirali AS, Bischoff MS, Lin HM, et al. Predicting the risk for acute type B aortic dissection in hypertensive patients using anatomic variables. JACC Cardiovasc Imaging. 2013;6(3):349-357. doi:10.1016/j.jcmg.2012.07.018

8. Meyrignac $\mathrm{O}, \mathrm{Bal} \mathrm{L}$, Zadro $\mathrm{C}$, et al. Combining volumetric and wall shear stress analysis from CT to assess risk of abdominal aortic aneurysm progression. Radiology. 2020;295(3):722-729. doi:10.1148/radiol.2020192112

9. Kumar A, Thompson EW, Lefieux A, et al. High coronary shear stress in patients with coronary artery disease predicts myocardial infarction. J Am Coll Cardiol. 2018;72(16):1926-1935. doi:10.1016/ j.jacc.2018.07.075

10. Gaur S, Taylor CA, Jensen JM, et al. FFR Derived from coronary CT angiography in nonculprit lesions of patients with recent STEMI JACC Cardiovasc Imaging. 2017;10(4):424-433. doi:10.1016/j. jcmg.2016.05.019

11. Shalhub S, Schafer M, Hatsukami TS, et al. Association of variant arch anatomy with type B aortic dissection and hemodynamic mechanisms. J Vasc Surg. 2018;68(6):1640-1648. doi:10.1016/j. jvs.2018.03.409

12. Ben Ahmed S, Dillon-Murphy D, Figueroa CA. Computational study of anatomical risk factors in idealized models of Type B aortic dissection. Eur J Vasc Endovasc Surg. 2016;52(6):736-745. doi:10.1016/j.ejvs.2016.07.025

13. Polanczyk A, Piechota-Polanczyk A, Domenig C, Nanobachvili J, Huk I, Neumayer C. Computational fluid dynamic accuracy in mimicking changes in blood hemodynamics in patients with acute Type IIIb aortic dissection treated with TEVAR. Applied Sciences. 2018;8:8. doi:10.3390/app8081309

14. Polanczyk A, Piechota-Polanczyk A, Stefanczyk L, Strzelecki M. Shape and enhancement analysis as a useful tool for the presentation of blood hemodynamic properties in the area of aortic dissection. J Clin Med. 2020;9:5. doi:10.3390/jcm9051330

15. Tse KM, Chiu P, Lee HP, Ho P. Investigation of hemodynamics in the development of dissecting aneurysm within patient-specific dissecting aneurismal aortas using computational fluid dynamics (CFD) simulations. $J$ Biomech. 2011;44(5):827-836. doi:10.1016/j. jbiomech.2010.12.014

16. Zhang X, Luo M, Fang K, et al. Analysis of the formation mechanism and occurrence possibility of Post-Stenotic Dilatation of the aorta by CFD approach. Comput Methods Programs Biomed. 2020;194:105522. doi:10.1016/j.cmpb.2020.105522

17. Ganten MK, Weber TF, von Tengg-kobligk H, et al. Motion characterization of aortic wall and intimal flap by ECG-gated CT in patients with chronic B-dissection. Eur $J$ Radiol. 2009;72 (1):146-153. doi:10.1016/j.ejrad.2008.06.024

18. Leuprecht A, Perktold K. Computer simulation of non-Newtonian effects on blood flow in large arteries. Comput Methods Biomech Biomed Engin. 2001;4(2):149-163. doi:10.1080/10255840008908002

19. Naim WNWA, Ganesan PB, Sun Z, et al. Prediction of thrombus formation using vortical structures presentation in Stanford type B aortic dissection: a preliminary study using CFD approach. Appl Math Model. 2016;40(4):3115-3127. doi:10.1016/j.apm.2015.09.096
20. Craiem D, El Batti S, Casciaro ME, et al. Age-related changes of thoracic aorta geometry used to predict the risk for acute type B dissection. Int $J$ Cardiol. 2017;228:654-660. doi:10.1016/j. ijcard.2016.11.125

21. Rylski B, Desjardins B, Moser W, Bavaria JE, Milewski RK. Genderrelated changes in aortic geometry throughout life. Eur J Cardiothorac Surg. 2014;45(5):805-811. doi:10.1093/ejcts/ezt597

22. Hiratzka LF, Bakris GL, Beckman JA, et al. 2010 ACCF/AHA/ AATS/ACR/ASA/SCA/SCAI/SIR/STS/SVM guidelines for the diagnosis and management of patients with Thoracic Aortic Disease: a report of the American College of Cardiology Foundation/ American Heart Association Task Force on Practice Guidelines, American Association for Thoracic Surgery, American College of Radiology, American Stroke Association, Society of Cardiovascular Anesthesiologists, Society for Cardiovascular Angiography and Interventions, Society of Interventional Radiology, Society of Thoracic Surgeons, and Society for Vascular Medicine. Circulation. 2010;121(13):e266-369. doi:10.1161/CIR.0b013e3181d4739e

23. den Hartog AW, de Witte P, Radonic T, et al. Aortic disease in patients with Marfan syndrome aortic volume assessment for surveillance. Radiology. 2013;269:370-377. doi:10.1148/ radiol.13122310/-/DC1

24. Trinh B, Dubin I, Rahman O, et al. Aortic volumetry at contrast-enhanced magnetic resonance angiography: feasibility as a sensitive method for monitoring bicuspid aortic valve aortopathy. Invest Radiol. 2017;52(4):216-222. doi:10.1097/RLI.000000000 0000332

25. Zhe Liu Y, Sa JO. Multilocus association mapping using generalized ridge logistic regression. BMC Bioinform. 2011;12:384-391. doi:10.1186/1471-2105-12-384

26. Arashi M, Roozbeh M, Hamzah NA, Gasparini M. Ridge regression and its applications in genetic studies. PLoS One. 2021;16(4): e0245376. doi:10.1371/journal.pone. 0245376

27. Wei M, Li C, Dai Y, et al. High-throughput absolute quantification sequencing revealed osteoporosis-related gut microbiota alterations in Han Chinese elderly. Front Cell Infect Microbiol. 2021;11:630372. doi:10.3389/fcimb.2021.630372

28. Ranjbar S, Sardari Nia P, Karvandi M, Maessen J. Computational fluid dynamics in aortic arch pathophysiology. Eur J Cardiothorac Surg. 2017;51(2):398. doi:10.1093/ejcts/ezw286

29. Sherifova S, Holzapfel GA. Biomechanics of aortic wall failure with a focus on dissection and aneurysm: a review. Acta Biomater. 2019;99:1-17. doi:10.1016/j.actbio.2019.08.017

30. Morris PD, Narracott A, von Tengg-kobligk H, et al. Computational fluid dynamics modelling in cardiovascular medicine. Heart. 2016;102(1):18-28. doi:10.1136/heartjnl-2015-308044

31. Suh GY, Les AS, Tenforde AS, et al. Quantification of particle residence time in abdominal aortic aneurysms using magnetic resonance imaging and computational fluid dynamics. Ann Biomed Eng. 2011;39(2):864-883. doi:10.1007/s10439-010-0202-4
International Journal of General Medicine

\section{Publish your work in this journal}

The International Journal of General Medicine is an international, peer-reviewed open-access journal that focuses on general and internal medicine, pathogenesis, epidemiology, diagnosis, monitoring and treatment protocols. The journal is characterized by the rapid reporting of reviews, original research and clinical studies across all disease areas. The manuscript management system is completely online and includes a very quick and fair peer-review system, which is all easy to use. Visit http://www.dovepress.com/ testimonials.php to read real quotes from published authors. 